\title{
Is Aspirin Still the Cornerstone of Antiplatelet Therapy in Patients With Coronary Artery Disease? An Historical and Practical Narrative Review
}

\author{
Carol Gravinese ${ }^{1}$, Matteo Bianco ${ }^{1 *}$, Enrico Cerrato ${ }^{2}$, Paola Destefanis ${ }^{1}$, Alessia Luciano ${ }^{1}$, Alessandro \\ Bernardi ${ }^{3}$, Simone Bellucca ${ }^{1}$, Ferdinando Varbella ${ }^{2}$, Fiorenzo Gaita ${ }^{3}$, Roberto Pozzi ${ }^{1}$ \\ 1'Division of Cardiology, A.O.U San Luigi Gonzaga, Orbassano, Torino, Italy \\ ${ }^{2}$ Interventional Cardiology Unit, Infermi Hospital, Rivoli and San Luigi Gonzaga University Hospital, Orbassano, Italy \\ ${ }^{3}$ Dipartimento di Scienze Mediche, Divisione di Cardiologia, Città della Salute e della Scienza, Turin, Italy
}

*Corresponding Author: Matteo Bianco, M.D., Division of Cardiology, A.O.U. San Luigi Gonzaga, S.C. Cardiologia, Regione Gonzole 10, 10043, Orbassano, Turin, Italy. Tel: +390119026848, Email: matteo.bianco87@gmail.com

Received May 25, 2017; Accepted July 31, 2017; Online Published August 22, 2017

\begin{abstract}
Aspirin is an irreversible and non-selective inhibitor of cyclo-oxygenase. It represents the cornerstone of antiplatelet therapy and is used in secondary prevention of cardiovascular disease. Disagreement over the optimal maintenance dosage still exists; in America and Europe the most used doses of aspirin are $81 \mathrm{mg}$ and $100 \mathrm{mg}$ daily, respectively. There is also debate on the formulation and route of administration of the loading dose. The latest studies advise chewable and non-enteric coated aspirin; intravenous administration represents an alternative for unconscious or shocked patients. Aspirin hypersensitivity is characterized by the onset of respiratory, mucocutaneous, and systemic symptoms. It is marginally considered, but its prevalence is significant. International cardiologic guidelines only report the possibility of desensitizing intolerant patients or, alternatively, administering one single antiplatelet agent. Desensitization can induce a temporary tolerance to the drug and consists of the administration of sequential and incremental doses of aspirin. Rapid desensitization protocols have proven to be safe and effective in the vast majority of cases, and they should be included in the management of these patients. New studies are being carried out comparing aspirin with other antiplatelet agents, and the results will be available shortly.

Keywords: Coronary Artery Disease/Therapy, Drug Therapy, Combination Drug-Eluting Stents, Platelet Aggregation Inhibitors/ Administration and Dosage, Platelet Aggregation Inhibitors/Adverse Effects, Percutaneous Coronary Intervention
\end{abstract}

\section{Introduction}

Aspirin is the most widely used drug in the world. ${ }^{1}$ This primacy is given to its ability to prevent the occurrence of cardiovascular disease through the inhibition of platelet aggregation. Salicylic acid, aspirin's main active metabolite, performs its action in several tissues determining an irreversible and non-selective inhibition of cyclooxygenase (COX) by inactivating both of its isoforms: the "constitutive" COX-1 and the "inducible" COX-2. These enzymes act on a common substrate, arachidonic acid, and produce some of the most important mediators in inflammatory response and platelet aggregation. Aspirin's mechanism of action involves a reduction in prostaglandins and thromboxane A2 levels, which is at the basis of its analgesic, anti-inflammatory, antipyretic, and antiaggregating effects ${ }^{2-4}$ (Figure 1). The aims of this narrative review are to summarize the foremost articles focusing on the role of aspirin in the cardiovascular setting and to introduce upcoming papers on this topic.

\section{Optimal Use of Aspirin in Secondary Prevention of Acute Coronary Syndromes}

The beneficial role of aspirin in the secondary prevention of cardiovascular disease has been reinforced by numerous randomized trials and meta-analyses. In particular, the Second International Study of Infarct Survival (ISIS-2), begun in 1988, analyzed the role of acetylsalicylic acid on the survival of patients with myocardial infarction treated with streptokinase and aspirin. After 30 days of treatment, these patients showed clear benefits in terms of survival, with 26 fewer deaths out of 1000 patients compared to the control population. The benefit was maintained in the following 10 years of follow-up. The dose tested in the study was $160 \mathrm{mg}$ orally. ${ }^{5}$

The evidence of the crucial role of aspirin in infarcted patients led to the multiplication of studies on this topic.

Copyright (C) 2017 The Author(s). This is an open-access article distributed under the terms of the Creative Commons Attribution License (http:// creativecommons.org/licenses/by/4.0), which permits unrestricted use, distribution, and reproduction in any medium, provided the original work is properly cited. 


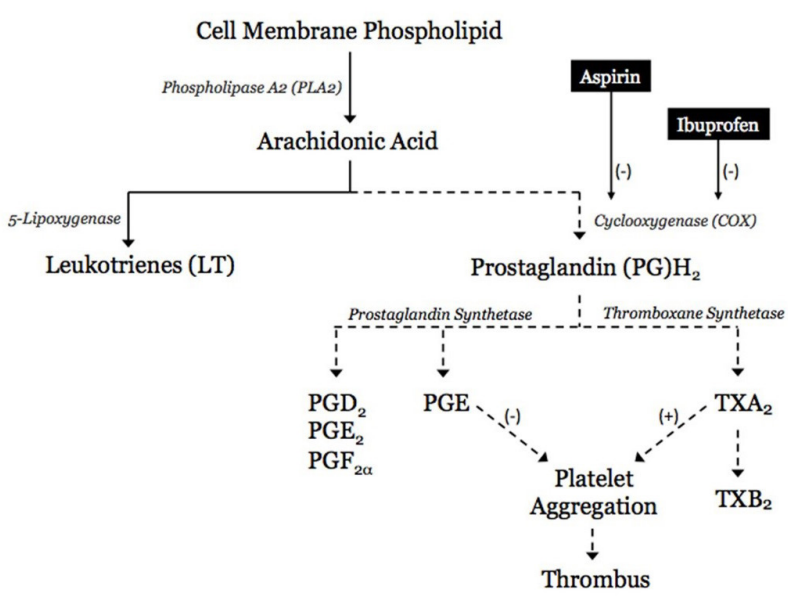

Figure 1. Aspirin Mechanism of Action.

TxA2: thromboxane A2. PG: prostaglandin. LT: leukotriene. 5-hydroperoxyeicosatetraenoic acid. FLAP: 5-lipoxygenase activating protein. 5-LO: 5-lipoxygenase.

An important focus was to identify the optimal dose of this drug. The study entitled "Double-dose versus standard-dose clopidogrel and high-dose versus low-dose aspirin in individuals undergoing percutaneous coronary intervention for acute coronary syndromes" (CURRENTOASIS 7) compared the efficacy and safety of 75-100 mg vs 300-325 mg of aspirin daily on 17000 patients undergoing coronary angioplasty. This study was the first to prove that low doses of aspirin overlapped high doses in death and cardiovascular events prevention and had a superior safety profile due to a lower incidence of haemorrhagic events. ${ }^{6}$ The lacking relationship between incidence of thrombotic events and different dosages of aspirin was later confirmed by a vast meta-analysis, the Antithombotic Trialists' Collaboration, which was carried out on more than 60 trials in patients taking aspirin in secondary prevention. It was shown that the "medium dose" aspirin (75-325 mg/d) had a similar effect compared to higher doses of aspirin and there was no difference between the two dosages in preventing cardiovascular events. ${ }^{7}$

The following studies investigated with a pharmacodynamic approach the optimal and minimal dose assuring the best compromise between COX-1 inhibition and fewer incidences of events. In patients with prior acute coronary syndrome (ACS), the optimal dose to prevent new events was $160 \mathrm{mg}$; the number of haemorrhagic events (including fatal ones) at this dosage was comparable to those caused by $80 \mathrm{mg}$ daily. ${ }^{8,9}$

Given all these findings, the optimal dosage of aspirin has not yet been clearly identified, even if a range of doses within which the efficacy and safety of aspirin was tested is known. The absence of an optimal dose explains the subtle differences between recommendations of European and American cardiologic societies.

As a matter of fact, 2014 ESC/EACTS Guidelines on myocardial revascularisation recommend 75-100 mg of aspirin daily as a maintenance dose in long-term secondary prevention. ${ }^{10} 2011 \mathrm{AHA} / \mathrm{ACC}$ Guidelines for percutaneous coronary intervention advise a maintenance dose between 81 and $325 \mathrm{mg}$ daily "indefinitely", while highlighting that the results of the CURRENT-OASIS 7 study mainly support the first dosage. ${ }^{11}$

The research of the optimal maintenance dose in ACS patients is still an unmet issue. ${ }^{12}$ Nevertheless, on the basis of the latest observational and randomized clinical trials in interventional cardiology, the dosages of $100 \mathrm{mg}$ and 81 $\mathrm{mg}$ are currently the most adopted and accepted ones in Europe and America, respectively. ${ }^{13-17}$

\section{Formulation and Route of Administration of the Loading Dose}

Patients who need coronary angioplasty or suffer from ACS must be given, if not already under treatment, an aspirin loading dose as soon as possible. ${ }^{10,11}$ Unfortunately, after more than 30 years of using acetylsalicylic acid in clinical practice, there still is no agreement on the optimal route of administration nor on the ideal formulation in this particular contest.

ESC guidelines on STEMI (ST-segment elevation myocardial infarction), NSTEMI (Non-ST-segment elevation myocardial infarction), and myocardial revascularization recommend $150-300 \mathrm{mg}$ orally as a loading dose or $80-150 \mathrm{mg}$ intravenously without specifying the formulation or route of administration. ${ }^{10}$ This is an important difference compared to the AHA/ ACC guidelines, which clearly specify the indications of "chewable" and "non-enteric coated" aspirin. ${ }^{11}$

Enteric-coated aspirin must initially be avoided because of a patient's reduced and delayed drug absorption, a condition known as aspirin pseudoresistance. ${ }^{18}$ The rationale for this formulation is to retard the release of active ingredients until their arrival in the small bowel, with a consequent reduction in gastric damage. However, there is scant evidence of a net benefit in terms of gastric safety between coated and conventional formulations, and it seems that the damage is only shifted to the small intestine. ${ }^{19}$ Grosser et al compared immediate release and enteric coated formulations of aspirin in 400 healthy volunteers. ${ }^{18}$ The study included people with a poor reaction to aspirin treatment, assessing it by serum $\mathrm{TxB} 2$ formation, an indicator of the ability of platelets to form COX-1-dependent TxA2-platelet aggregation ex vivo, and the urinary excretion of the thromboxane metabolite 11-dehydro TxB2 (TxM), an indicator of concrete thromboxane biosynthesis. Pseudoresistance was not assessable after the use of immediate release formulations, but was assessable after enteric coated ones and seemed more common after the latter when measurements were done 4 hours rather than 8 hours after dosing. Reinforcing the idea that pseudoresistance was widely ascribable to mutable aspirin exposure in individuals receiving enteric coated drug, there was a correction of phenotype in 101 of 108 patients. 
Today, few studies compare the differences in plasmatic concentration and platelet inhibition after various loading sources, and no study has yet investigated the prognostic impact of different formulations.

ISIS-2 considered fragmented or chewable formulations with a loading dose of $160 \mathrm{mg}$; AHA/ACC recommendations are based on these results. ${ }^{5}$

Two recent studies investigated the pharmacokinetics of chewable aspirin. Hobl et al examined the effects on healthy volunteers of various concentrations of acetylsalicylic acid and its metabolites and platelet inhibition levels after $162 \mathrm{mg}$ of chewable aspirin followed by $250 \mathrm{~mL}$ of water. Results showed that absorption was rapid and the maximum plasmatic concentration (Tmax) was reached in 30 minutes. Inhibition of platelet aggregation, measured with impedance aggregometry, was similar to that of acetylsalicylic acid (and its metabolite salicylic acid) concentration: $90 \%$ of platelets was inactivated after 30 minutes. $^{20}$

Nordt et al compared 3 different aspirin formulations on healthy volunteers: (1) solid aspirin tablet swallowed whole; (2) solid aspirin tablet chewed then swallowed; and (3) chewable aspirin formulation chewed and swallowed. Serum salicylate measurements were obtained over a 180-minute period. Results demonstrated that the fastest absorption rate was obtained with the chewable aspirin formulation, since initial serum salicylate concentrations were seen at different time points: 76 (solid aspirin tablet swallowed whole), 37 (solid aspirin tablet chewed then swallowed) and 21 (chewable aspirin formulation chewed and swallowed) minutes; moreover, the chewable formulation absorption was more complete than the other formulations at 180 minutes. ${ }^{21}$ These results indicate that in treating ACS, it may be advisable to use a chewable aspirin formulation rather than solid tablet aspirin either chewed or swallowed; however, this strategy needs validation with randomized trials not only on healthy patients, but also on ACS patients.

The intravenous route represents an alternative that ESC guidelines particularly recommend in unconscious patients or in patients who have difficulty taking the drug orally. Acetylsalicylic acid is not soluble in water and therefore is not administrable intravenously. To solve this problem, it is possible to use lysine acetylsalicylate, which is rapidly metabolized into acetylsalicylic acid in the stomach. Lysine acetylsalicylate is a drug commonly used in the cardiology setting, but there are no trials attesting to its efficacy and safety. The ECCLIPSE, a randomized cross-over trial, is the only work to test lysine acetylsalicylate in healthy volunteers. It compared a loading dose of $325 \mathrm{mg}$ of aspirin orally with a dose of $425 \mathrm{mg}$ of lysine acetylsalicylate intravenously, both associated with a loading dose of prasugrel. Inhibition of platelet aggregation was evaluated with aggregometry and found to be significantly superior in patients treated with lysine acetylsalicylate after only 30 minutes $(85.3 \% \pm 7.2 \%$ versus $44.3 \% \pm 32.6 \%, P=0.003)$. This difference was maintained and later progressively reduced until the twelfth hour after administration. ${ }^{22}$

In conclusion, further investigations are needed to identify the ideal formulation and administration route of aspirin in patients with ACS. The available data shows that a faster inhibition of platelet activity can be obtained through simple measures, such as chewing the loading dose or administering it intravenously. The latter, assuring a faster and more complete platelet inhibition, should be the first choice in unconscious patients, cases of shock, and recurrent emesis.

\section{Management of Aspirin-Intolerant Patients}

Aspirin is the second drug (after penicillin) that most frequently causes intolerance reactions. ${ }^{23}$ The fact that it is also the most prescribed drug in the world makes hypersensitivity to this drug a crucial topic, and the problem is additionally emphasized if these patients suffer from ACS. This observation comes from the fact that hypersensitivity precludes the possibility to benefit from a life-saving drug whose critical role was described in the previous chapters.

Unfortunately, international guidelines marginally deal with aspirin hypersensitivity management and only report the possibility to desensitize intolerant patients or, alternatively, administer one single antiplatelet agent. ${ }^{10,11}$

The National Institute for Clinical Excellence (NICE) in the United Kingdom has defined aspirin intolerance as "either a proven hypersensitivity to aspirin or a history of severe indigestion caused by low-dose aspirin." ${ }^{24}$

Aspirin intolerance has a variable prevalence depending on the population in study. It ranges between $0.2 \%$ in patients with chronic urticaria exacerbated by acetylsalicylic acid and $10 \%$ in people with allergic asthma. Although the exact prevalence is not definable, a recent survey on this topic showed aspirin hypersensitivity involves less than 5\% of patients undergoing PCI. This data has been confirmed by many observational studies, which have revealed a prevalence between $2.6 \%$ and $3.1 \%{ }^{25}$

The mechanism underlying aspirin hypersensitivity reactions can be either immunological or pharmacological; however, some patients could have both reactions. Allergic/immunological reactions are due to a specific immunologlobulin E (IgE) produced against the drug, whereas pharmacological reactions are mediated by inhibition of the COX-1 pathway. ${ }^{26}$ This represents the way to differentiate between anaphylactic and anaphylactoid reactions. ${ }^{27}$ The former is IgE mediated, while the latter is not (although it may be similar to anaphylactic symptoms). In addition, it may happen in the same patient that aspirin could trigger an immunological reaction at one time and a pharmacological reaction at another. ${ }^{28}$

Regarding the pharmacological reaction, aspirin hypersensitivity is probably due to an imbalance in arachidonic acid depletion induced by a sudden inhibition of COX in tissues. This inactivation leads to the loss of production of several cytokines, such as prostaglandin E2 (PGE2), which is an important mediator of inflammatory 
response through inhibition of mastocyte degranulation and lypo-oxygenase, an enzyme responsible for leukotrienes production. The lacking PGE2 production determines in particular patients a loss of regulation of inflammatory response and the onset of a clinical picture that resemble an allergic reaction. This scenario can have different degrees of severity and is characterized by respiratory (allergic rhinitis and asthma), cutaneous (urticaria and angioedema), and, more rarely, anaphylactoid (glottis edema) reactions. Patients with gastrointestinal problems, epistasis, or minor bleeding related to aspirin administration must not be considered intolerant patients as these symptoms are the most common adverse effects of the drug. ${ }^{29}$

The classification of Gollapudi et al includes 5 different types of aspirin intolerance reactions. ${ }^{30}$ Types $1,2,3$ are COX mediated and characterized by the presence of cross-reactivity with other NSAIDs. Type 2 is frequent in patients with chronic urticaria and is the most difficult one to desensitize; people with this intolerance are frequently excluded from desensitization protocols. However, recent data has shown that a dose-dependent effect of aspirin and tolerance to low doses of acetylsalicylic acid sufficient to achieve an antiaggregating effect can be obtained in these patients too. Types 4 and 5 are IgE mediated and do not have cross-reactivity with other nonsteroidal anti-inflammatory drugs (NSAIDs); some studies have reported the possibility of desensitizing these patients, although, because of the severity of hypersensitivity reactions (i.e. Stevens-Johnson syndrome and Lyell syndrome), the management should be very cautious along with the clinical support of an allergist (Table 1).

The optimal treatment for patients with aspirin hypersensitivity remains a challenge for the cardiologist. As stated before, guidelines do not provide clear recommendations for the management of aspirin intolerance despite numerous reports suggesting that rapid desensitization protocols are safe and effective. In addition, alternative strategies, such as an antiplatelet monotherapy with clopidogrel or the introduction of other drugs (e.g., indobufene), were proven to be respectively ineffective or tested on a number of patients too limited to represent a valid approach of evidence-based medicine. ${ }^{31}$

Desensitization for drug allergy has been defined as "the induction of temporary clinical unresponsiveness to drug antigens," ${ }^{26}$ or alternatively, "the elimination of pharmacological and immunological reactions by slowly increasing exposure to the drug." 28

The mechanism for aspirin desensitization is not yet completely understood, but it is believed that small incremental dosages downregulate cystienyl leukotriene receptors and reduce leukotriene production and histamine and tryptase release from mast cells. ${ }^{26,30-32}$

The mechanism underlying desensitization in individuals with IgE-mediated reactions is still unclear, but it is believed to be similar to penicillin desensitization, because there is also here the sporadic occurrence of $\operatorname{IgE}$ antibodies and the necessity of a previous contact. ${ }^{30}$ Moreover, a continuous NSAID exposure results in the saturation of anti-NSAID IgE antibody sites on basophils and mast cells. ${ }^{33}$ Additionally, there is a reduced activation of basophils and mast cells because of IgE cross-linking. Finally, sustained treatment with NSAID determines a continuing reduction of intracellular mediators, such as histamine.

Aspirin desensitization was initially used by pulmonologists to treat asthma induced by NSAIDs. Protocols consisted of sequential incremental doses until tolerance was achieved. In a cardiology setting, the main limits of these protocols are their long duration (generally 4-5 days) and the need to confirm the diagnosis with an aspirin challenge in order to verify the presence of intolerance reactions.

The need to obtain a rapid inhibition of platelet activity and the impossibility of exposing potentially unstable patients to the risk of hypersensitivity reactions administering a testing dose of aspirin led to the elaboration of rapid desensitization protocols. Since 2000, several protocols have been proposed, and they are synthetized

Table 1. Classification of Aspirin Intolerance Reactions ${ }^{30}$

\begin{tabular}{|c|c|c|c|c|}
\hline $\begin{array}{l}\text { Type of } \\
\text { Intolerance }\end{array}$ & Clinical Characteristics & $\begin{array}{l}\text { Cross Reactivity With } \\
\text { Other COX-1 Inhibitors }\end{array}$ & $\begin{array}{l}\text { Possibility of } \\
\text { Desensitization }\end{array}$ & Additional Characteristics \\
\hline Type 1 & $\begin{array}{l}\text { Chronic sinusitis and/or nasal } \\
\text { polyposis and asthma }\end{array}$ & Yes & Yes & $\begin{array}{l}\text { Easily obtainable desensitization even } \\
\text { at high dosages ( } 500 \mathrm{mg})\end{array}$ \\
\hline Type 2 & Chronic spontaneous urticarial & Yes & Difficult & $\begin{array}{l}\text { Desensitization obtainable only at low } \\
\text { doses }(<100 \mathrm{mg})\end{array}$ \\
\hline Type 3 & Urticaria, angioedema & Yes & Yes & $\begin{array}{l}\text { Usable in cases of intolerance to an } \\
\text { NSAID that is not ASA }\end{array}$ \\
\hline Type 4 & $\begin{array}{l}\text { Pseudoallergic reaction to a } \\
\text { specific NSAID }\end{array}$ & No & No & $\begin{array}{l}\text { Usable in cases of intolerance to an } \\
\text { NSAID that is not ASA }\end{array}$ \\
\hline Type 5 & $\begin{array}{l}\text { Fixed erythema, aseptic } \\
\text { meningitis, interstitial nephritis }\end{array}$ & No & No & $\begin{array}{l}\text { Steven-Johnson Syndrome, Lyell } \\
\text { Syndrome. Absolute contraindication } \\
\text { to ASA }\end{array}$ \\
\hline
\end{tabular}

Abbreviations: NSAID, non-steroidal anti-inflammatory drug; ASA, acetylsalicylic acid. 
in Table 2. The peculiarity of rapid desensitization is the possibility of achieving a tolerance to acetylsalicylic acid in 2 to 5 hours. $^{37}$

A recent meta-analysis compared different protocols enlightening their high safety profile and efficacy; desensitization was also performed in ACS patients, who represented $55 \%$ of the population. ${ }^{45}$ This work compared the intravenous desensitization protocol to the oral protocol: desensitization was successful in almost all patients (98\%, 95.9\% and 95.8\%, respectively). Incidence of urticaria, angioedema, and asthma during desensitization was less than $5 \%$ in all studies considered, and in most cases patients were desensitized restarting with the last tolerated dose before treatment interruption. More recent studies investigated the use of leukotriene inhibitors such as montelukast and antihistamines as a pretreatment, but the results were superimposable. There is little data analyzing aspirin hypersensitivity in STEMI patients, but some case series showed the possibility of undergoing PCI with a P2Y12 inhibitor and a GP IIb/IIIa receptor inhibitor, desensitizing the patient 24 hours after the procedure. Once the desensitization protocol is completed, it is important to keep on assuming aspirin and not to interrupt it for more than 5 days in a row; otherwise, the upregulation of leukotriene receptors will make another desensitization procedure necessary (Table 2). ${ }^{25}$

The ADAPTED Registry was the largest study exploring the systematic use of ASA desensitization in a large cohort of patients with $\mathrm{CAD}$, both acute and stable, undergoing invasive evaluation with the intent to undergo PCI. A total of 330 patients were enrolled, including high coronary risk patients, such as patients with STEMI, and those who had a history of severe anaphylactic reactions, such as anaphylactic shock. Results showed that a 6-step oral protocol carried out over 5.5 hours was safe, as no severe hypersensitivity reaction occurred, and effective in a wide cohort of non-selected patients with a success rate of $95.4 \%{ }^{46}$

Focusing on other drug interactions, there could be an increased sensitivity to allergens associated with therapy with beta-blockers, which could culminate in a dangerous hypersensitivity reaction. ${ }^{47-49}$ In addition, beta-blockers may cause a reduction in the adrenaline effect, which represents a fundamental drug in the treatment of serious hypersensitivity responses. ${ }^{47,50}$ It is thus advisable to suspend beta-blockers 24 hours before aspirin desensitization, although individuals who experience symptoms of coronary disease need to be evaluated singularly. ${ }^{50}$

ACE inhibitor treatment in individuals with a history of angioedema unrelated to this drug could expose them to a higher possibility of angioedema while assuming an ACE inhibitor; therefore, in patients with hereditary or idiopathic angioedema, therapy with ACE inhibitors should be assessed cautiously or avoided. ${ }^{47,51}$ Treatment with ACE inhibitors while the desensitization procedure was performed caused persistent systemic responses; in these patients, there was no reaction when ACE inhibitors were momentarily stopped, but symptoms returned in cases of unintentional re-exposure. On the basis of this data, it may be advisable to discontinue ACE inhibitors before desensitization procedures to avoid systemic responses; in this setting, angiotensin receptor blockers may represent a valid substitute.

\section{Recommendations for the Future}

The future of aspirin in ACS appears to be guaranteed by the huge amount of data in its favor. ${ }^{52}$ In addition to the protective cardiovascular effect, there is consistent data supporting the idea of aspirin involvement in terms of mortality, incidence of the main solid tumors (colorectal

Table 2. Aspirin Desensitization Protocols

\begin{tabular}{|c|c|c|c|}
\hline Protocol & Dose (mg) & Duration (h) & Other Drugs Administered \\
\hline \multicolumn{4}{|c|}{ Intravenous } \\
\hline De Luca, $2013^{34}$ & $1,2,4,8,16,32,64,128,250$ & 4.5 & No \\
\hline \multicolumn{4}{|c|}{ Oral, Less Than 6 Steps } \\
\hline Cortellini, low risk $2012^{35}$ & $10,15,25,20,50$ & 3 & No \\
\hline Lee, $2013^{36}$ & $5,10,20,40,80$ & 3 & No \\
\hline Rossini, $2008^{37}$ & $1,15,10,20,40,100$ & 5.5 & No \\
\hline Silberman, $2004^{38}$ & $5,10,20,40,75$ & 2.5 & No \\
\hline Veas, $2013^{39}$ & $1,5,10,20,40,100$ & & \\
\hline \multicolumn{4}{|c|}{ Oral, More Than 6 Steps } \\
\hline Christou, $2011^{40}$ & $0.1,0.3,10,30,40,81,162,325$ & 3.5 & No \\
\hline Cortellini, low risk $2012 \frac{35}{5}$ & $0,1,1,1,5,2,3,4,5,10,15,25,35,50$ & 3.5 & No \\
\hline Dalmau, $2009^{41}$ & $0.1,0.2,1,3,10,25,50,100$ & 2.5 & No \\
\hline Wong, $1999^{42}$ & $0.1,0.3,1,3,10,20,40,81,162,325$ & 3.5 & Antihistamine \\
\hline McMullan, $2013^{43}$ & $1,10,20,40,80,160,325$ & 2 & No \\
\hline Cordoba-Soriano, $2015^{44}$ & $0.1,0.3,1,3,10,25,50,100$ & 1.75 & Antihistamine, corticosteroid, or antileukotriene \\
\hline
\end{tabular}


cancer), and metastatic dissemination. These results are considered in the 2016 US Preventive Services Task Force (USPSTF) guidelines. ${ }^{53-57}$

The WOEST randomized trial recently compared a double therapy with clopidogrel and warfarin vs. a triple therapy with aspirin, clopidogrel, and warfarin in patients undergoing coronary angioplasty. The trial revealed a smaller incidence of bleeds in the double therapy group compared to the triple therapy one, without an increase in secondary endpoints, i.e. death, myocardial infarction, revascularization, stroke, or stent thrombosis. This work was criticized because of the scant sample size (573 patients), the high incidence of minor bleeds (partly due to inadequate gastroprotective therapy), the absence of an aspirin-warfarin group, and a statistical power sufficient to elucidate significant differences in thrombosis. ${ }^{57}$ Despite these limits, the latest ESC NSTEMI guidelines recommend, after 6 months of triple therapy, 1-year monotherapy with an antiplatelet agent associated with an anticoagulant in patients undergoing PCI with low bleeding risk. The antiplatelet agent to be used is chosen by cardiologists and may not include aspirin. After one year, patients continue the treatment with the anticoagulant alone. In patients at high risk of bleeding with HAS-BLED $\geq 3$, triple therapy is only recommended for 30 days and can be substituted with a double therapy consisting of clopidogrel/ASA and warfarin (class of recommendation: IIb)..$^{58,59}$

Thanks to a widely-documented efficacy and an optimal cost/benefit relationship, in the absence of contraindications, chronic and limitless ASA therapy for secondary prevention of ischemic events continues to be recommended by the latest European guidelines (class I, level A). The search for alternatives to aspirin in this setting is currently a vivid investigation topic. A well-known CAPRIE trial which compared aspirin and clopidogrel revealed a slight superiority with the latter, driven by its effect on arterial disease; concerning post infarction and post stroke, the comparison showed an absolute similarity between the two drugs. ${ }^{60}$

A recent work that recruited more than 3000 patients investigated the maintenance of clopidogrel after the twelfth month of dual antiplatelet therapy (DAPT) instead of aspirin. After a 3-year follow-up, results demonstrated that the group treated with clopidogrel had a reduction in cardiovascular death, myocardial infarction, and stroke without an increase in major bleeds.

The study GLOBAL LEADERS: A Clinical Study Comparing Two Forms of Anti-platelet Therapy After Stent Implantation (NCT01813435), currently ongoing, is exploring a strategy of early interruption of DAPT with aspirin and ticagrelor vs. standard therapy; the group that interrupts DAPT earlier is treated with ticagrelor monotherapy for 23 months. This work will provide further information about the possibility for ticagrelor to challenge aspirin's throne in long-term cardiovascular prevention or will confirm a similarity in preventive effects, as The Acute

\section{Review Highlights}

\section{What Is Already Known?}

Aspirin is an irreversible and non-selective inhibitor of cyclo-oxygenase and represents the cornerstone of anti-aggregating therapy. Several studies have proven its efficacy in reducing platelet activity and its irreplaceability in DAPT.

\section{What This Study Adds?}

Rapid desensitization protocols have proven to be safe and effective in the vast majority of cases, and they should be included in the management of cardiology patients. Further studies are needed to assess whether aspirin is always necessary or new antiplatelet agents can be substituted for it.

Stroke or Transient Ischaemic Attack Treated with Aspirin or Ticagrelor and Patient Outcomes (SOCRATES) study ${ }^{61}$ already showed in the cerebrovascular area. Indeed, the SOCRATES trial was the first to compare ticagrelor and aspirin in a 90-day prevention of major cardiovascular events (stroke, myocardial infarction, death) in post stroke/TIA patients. Loading doses of $180 \mathrm{mg}$ of ticagrelor and $300 \mathrm{mg}$ of aspirin were followed by ticagrelor $90 \mathrm{mg}$ bid and aspirin $100 \mathrm{mg}$. Ticagrelor showed no significant superiority in terms of preventive efficacy compared to aspirin, with increased bleeds and superimposable tolerability.

\section{Conclusion}

Aspirin is used in the secondary prevention of ACS. The most common dosages in Europe and America are $100 \mathrm{mg}$ and $81 \mathrm{mg}$, respectively. Chewable and non-enteric coated aspirin should be preferred over solid tablets; intravenous administration represents a valid alternative, in particular in unconscious patients, shock, or recurrent emesis.

Aspirin hypersensitivity is an underestimated issue, but it must be taken into account because of its implications on clinical practice. Aspirin desensitization represents a safe and effective opportunity to benefit from this life-saving drug through the administration of incremental and sequential doses of aspirin.

Challenges between the cornerstone of cardiovascular prevention and new antiplatelet agents (ticagrelor primarily) are still ongoing; interesting therapeutic indications for the future will be arriving shortly.

\section{Authors' Contributions}

All authors contributed equally to this research.

\section{Conflict of Interest Disclosures}

None of the authors involved in the study have any conflicts of interest or disclosures related to the topic of the study.

\section{Ethical Approval}

Not applicable. 


\section{Acknowledgments}

The authors wish to thank Michael Andrews for his valuable contribution to the English revision.

\section{References}

1. Vane JR, Botting RM. The mechanism of action of aspirin. Thromb Res. 2003;110(5-6):255-258. doi:10.1016/S00493848(03)00379-7.

2. Vane JR, Botting RM. Aspirin and Other Salicylates. London: Chapman \& Hall Medical; 1992.

3. Gilroy DW. The role of aspirin-triggered lipoxins in the mechanism of action of aspirin. Prostaglandins Leukot Essent Fatty Acids. 2005;73(3-4):203-10. doi:10.1016/j. plefa.2005.05.007

4. Katzung BG. Basic \& Clinical Pharmacology. 10th ed. New York: McGraw-Hill Professional; 2006.

5. Baigent C, Collins R, Appleby P, Parish S, Sleight P, Peto R. ISIS-2: 10 year survival among patients with suspected acute myocardial infarction in randomised comparison of intravenous streptokinase, oral aspirin, both, or neither. The ISIS-2 (Second International Study of Infarct Survival) Collaborative Group. BMJ. 1998;316(7141):1337-1343. doi:10.1136/ bmj.316.7141.1337.

6. Mehta SR, Tanguay JF, Eikelboom JW, et al. Double-dose versus standard-dose clopidogrel and high-dose versus lowdose aspirin in individuals undergoing percutaneous coronary intervention for acute coronary syndromes (CURRENT-OASIS 7): a randomised factorial trial. Lancet. 2010;376(9748):12331243. doi:10.1016/s0140-6736(10)61088-4.

7. Collaborative overview of randomised trials of antiplatelet therapy--I: Prevention of death, myocardial infarction, and stroke by prolonged antiplatelet therapy in various categories of patients. Antiplatelet Trialists' Collaboration. BMJ. 1994;308(6921):81-106. doi:10.1136/bmj.308.6921.81.

8. Dalen JE. Aspirin to prevent heart attack and stroke: what's the right dose? Am J Med. 2006;119(3):198-202. doi:10.1016/j. amjmed.2005.11.013.

9. DiNicolantonio JJ, Norgard NB, Meier P, et al. Optimal aspirin dose in acute coronary syndromes: an emerging consensus. Future Cardiol. 2014;10(2):291-300. doi:10.2217/fca.14.7.

10. Windecker S, Kolh P, Alfonso F, et al. 2014 ESC/EACTS Guidelines on myocardial revascularization: The Task Force on Myocardial Revascularization of the European Society of Cardiology (ESC) and the European Association for Cardio-Thoracic Surgery (EACTS) Developed with the special contribution of the European Association of Percutaneous Cardiovascular Interventions (EAPCI). Eur Heart J. 2014;35(37):2541-2619. doi:10.1093/eurheartj/ehu278.

11. Levine GN, Bates ER, Blankenship JC, et al. 2011 ACCF/AHA/ SCAI Guideline for Percutaneous Coronary Intervention. A report of the American College of Cardiology Foundation/ American Heart Association Task Force on Practice Guidelines and the Society for Cardiovascular Angiography and Interventions. J Am Coll Cardiol. 2011;58(24):e44-e122. doi:10.1016/j.jacc.2011.08.007.

12. Bem D, Dretzke J, Stevens S, et al. Investigating the effectiveness of different aspirin dosing regimens and the timing of aspirin intake in primary and secondary prevention of cardiovascular disease: protocol for a systematic review. Syst Rev. 2015;4:88. doi:10.1186/s13643-015-0078-3.

13. Biondi-Zoccai G, Lotrionte M, Agostoni P, et al. Adjusted indirect comparison meta-analysis of prasugrel versus ticagrelor for patients with acute coronary syndromes. Int J Cardiol. 2011;150(3):325-331. doi:10.1016/j.ijcard.2010.08.035.

14. D'Ascenzo F, Barbero U, Bisi M, et al. The prognostic impact of high on-treatment platelet reactivity with aspirin or ADP receptor antagonists: systematic review and meta-analysis. Biomed Res Int. 2014;2014:610296. doi:10.1155/2014/610296.

15. D'Ascenzo F, Taha S, Moretti C, et al. Meta-analysis of randomized controlled trials and adjusted observational results of use of clopidogrel, aspirin, and oral anticoagulants in patients undergoing percutaneous coronary intervention. Am J Cardiol. 2015;115(9):1185-1193. doi:10.1016/j.amjcard.2015.02.003.

16. Bollati M, Gaita F, Anselmino M. Antiplatelet combinations for prevention of atherothrombotic events. Vasc Health Risk Manag. 2011;7:23-30. doi:10.2147/vhrm.s12271.

17. D'Ascenzo F, Colombo F, Barbero $U$, et al. Discontinuation of dual antiplatelet therapy over 12 months after acute coronary syndromes increases risk for adverse events in patients treated with percutaneous coronary intervention: systematic review and meta-analysis. J Interv Cardiol. 2014;27(3):233-241. doi:10.1111/joic.12107

18. Grosser T, Fries S, Lawson JA, Kapoor SC, Grant GR, FitzGerald GA. Drug resistance and pseudoresistance: an unintended consequence of enteric coating aspirin. Circulation. 2013;127(3):377-385. doi:10.1161/circulationaha.112.117283

19. Endo H, Sakai E, Higurashi T, et al. Differences in the severity of small bowel mucosal injury based on the type of aspirin as evaluated by capsule endoscopy. Dig Liver Dis. 2012;44(10):833-838. doi:10.1016/j.dld.2012.05.016.

20. Hobl EL, Schmid RW, Stimpfl T, Ebner J, Jilma B. Absorption kinetics of low-dose chewable aspirin--implications for acute coronary syndromes. Eur J Clin Invest. 2015;45(1):13-17. doi:10.1111/eci.12373.

21. Nordt SP, Clark RF, Castillo EM, Guss DA. Comparison of three aspirin formulations in human volunteers. West J Emerg Med. 2011;12(4):381-385. doi:10.5811/westjem.2011.4.2222.

22. Vivas D, Martin A, Bernardo E, et al. Impact of intravenous lysine acetylsalicylate versus oral aspirin on prasugrel-inhibited platelets: results of a prospective, randomized, crossover study (the ECCLIPSE Trial). Circ Cardiovasc Interv. 2015;8(5). doi:10.1161/circinterventions.114.002281.

23. Ganjehei L, Becker RC. Aspirin dosing in cardiovascular disease prevention and management: an update. J Thromb Thrombolysis. 2015;40(4):499-511. doi:10.1007/s11239-0151267-6

24. National Institute for Health and Clinical Excellence. Clopidogrel and modified release dipyridamole in the prevention of occlusive vascular events. Published 2005.

25. Bianco M, Bernardi A, D'Ascenzo F, et al. Efficacy and safety of available protocols for aspirin hypersensitivity for patients undergoing percutaneous coronary intervention: a survey and systematic review. Circ Cardiovasc Interv. 2016;9(1):e002896. doi:10.1161/circinterventions.115.002896.

26. Castells M. Desensitization for drug allergy. Curr Opin Allergy Clin Immunol. 2006;6(6):476-481. doi:10.1097/ ACl.0b013e3280108716.

27. Lambrakis P, Rushworth GF, Adamson J, Leslie SJ. Aspirin hypersensitivity and desensitization protocols: implications for cardiac patients. Ther Adv Drug Saf. 2011;2(6):263-270. doi:10.1177/2042098611422558.

28. Silberman S, Neukirch-Stoop C, Steg PG. Rapid desensitization procedure for patients with aspirin hypersensitivity undergoing coronary stenting. Am J Cardiol. 2005;95(4):509-510. doi:10.1016/j.amjcard.2004.10.022.

29. Jenneck C, Juergens U, Buecheler M, Novak N. Pathogenesis, diagnosis, and treatment of aspirin intolerance. Ann Allergy Asthma Immunol. 2007;99(1):13-21. doi:10.1016/s10811206(10)60615-1.

30. Gollapudi RR, Teirstein PS, Stevenson DD, Simon RA. Aspirin sensitivity: implications for patients with coronary artery disease. JAMA. 2004;292(24):3017-3023. doi:10.1001/ jama.292.24.3017. 
31. Ramanuja S, Breall JA, Kalaria VG. Approach to "aspirin allergy" in cardiovascular patients. Circulation. 2004;110(1):e1-e4. doi:10.1161/01.cir.0000134306.28561.2a.

32. Stevenson DD, SzczeklikA. Clinical and pathologic perspectives on aspirin sensitivity and asthma. J Allergy Clin Immunol. 2006;118(4):773-786. doi:10.1016/j.jaci.2006.07.024.

33. Solensky R. Drug desensitization. Immunol Allergy Clin North Am. 2004;24(3):425-443. doi:10.1016/j.iac.2004.03.008.

34. De Luca G, Verdoia M, Binda G, Schaffer A, Suryapranata $\mathrm{H}$, Marino P. Aspirin desensitization in patients undergoing planned or urgent coronary stent implantation. A single-center experience. Int J Cardiol. 2013;167(2):561-563. doi:10.1016/j. ijcard.2012.01.063.

35. Cortellini G, Testi S, Severino M, et al. Aspirin challenge/ desensitisation before coronary stenting in subjects with history of hypersensitivity. A pragmatic approach. Eur Ann Allergy Clin Immunol. 2012;44(4):160-162.

36. Lee JK, Tsui KL, Cheung CY, et al. Aspirin desensitisation for Chinese patients with coronary artery disease. Hong Kong Med J. 2013;19(3):207-213. doi:10.12809/hkmj133914.

37. Rossini R, Angiolillo DJ, Musumeci G, et al. Aspirin desensitization in patients undergoing percutaneous coronary interventions with stent implantation. Am J Cardiol. 2008;101(6):786-789. doi:10.1016/j.amjcard.2007.10.045.

38. Silberman S, Neukirch-Stoop C, Steg PG. Rapid desensitization procedure for patients with aspirin hypersensitivity undergoing coronary stenting. Am J Cardiol. 2005;95(4):509-510. doi:10.1016/j.amjcard.2004.10.022.

39. Veas PN, Martinez G, Jalil MJ, Martinez SA, Castro GP. [Rapid aspirin desensitization in patients with a history of aspirin hypersensitivity requiring coronary angioplasty. Report of four cases]. Rev Med Chil. 2013;141(2):255-259. doi:10.4067/ s0034-98872013000200016.

40. Christou A, Kafkas N, Marinakos A, Katsanos S, Papanikitas K, Patsilinakos S. Rapid desensitisation of patients with aspirin allergy who undergo coronary angioplasty. Hellenic J Cardiol. 2011;52(4):307-310.

41. Dalmau G, Gaig P, Gazquez V, Merce J. Rapid desensitization to acetylsalicylic acid in acute coronary syndrome patients with NSAID intolerance. Rev Esp Cardiol. 2009;62(2):224-225.

42. Wong JT, Nagy CS, Krinzman SJ, Maclean JA, Bloch KJ. Rapid oral challenge-desensitization for patients with aspirin-related urticaria-angioedema. J Allergy Clin Immunol. 2000;105(5):9971001. doi:10.1067/mai.2000.104571.

43. McMullan KL, Wedner HJ. Safety of aspirin desensitization in patients with reported aspirin allergy and cardiovascular disease. Clin Cardiol. 2013;36(1):25-30. doi:10.1002/ clc.22054.

44. Cordoba-Soriano JG, Corbi-Pascual M, Lopez-Neyra I, Navarro-Cuartero J, Hidalgo-Olivares V, Barrionuevo-Sanchez $\mathrm{MI}$, et al. Early aspirin desensitization in unstable patients with acute coronary syndrome: Short and long-term efficacy and safety. Eur Heart J Acute Cardiovasc Care. 2016;5(7):41-50. doi:10.1177/2048872615618509.

45. D'Ascenzo F, Moretti C, Bianco M, et al. Meta-analysis of the duration of dual antiplatelet therapy in patients treated with second-generation drug-eluting stents. Am J Cardiol. 2016;117(11):1714-1723. doi:10.1016/j. amjcard.2016.03.005.

46. Rossini R, lorio A, Pozzi R, et al. Aspirin desensitization in patients with coronary artery disease: results of the multicenter ADAPTED Registry (Aspirin Desensitization in Patients With Coronary Artery Disease). Circ Cardiovasc Interv. 2017;10(2). doi:10.1161/circinterventions.116.004368.

47. Sweetman SC. Martindale: The Complete Drug Reference. 36th ed. London: Pharmaceutical Press; 2009.

48. Lang DM. Do beta-blockers really enhance the risk of anaphylaxis during immunotherapy? Curr Allergy Asthma Rep. 2008;8(1):37-44. doi:10.1007/s11882-008-0008-8.

49. TenBrook JAJr, Wolf MP, Hoffman SN, etal. Should beta-blockers be given to patients with heart disease and peanut-induced anaphylaxis? A decision analysis. J Allergy Clin Immunol. 2004;113(5):977-982. doi:10.1016/j.jaci.2004.02.043.

50. Soar J, Pumphrey R, Cant A, et al. Emergency treatment of anaphylactic reactions--guidelines for healthcare providers. Resuscitation. 2008;77(2):157-169. doi:10.1016/j. resuscitation.2008.02.001.

51. AstraZeneca UK Ltd. Zestril tablets: summary of product characteristics. http://www.medicines.ie/medicine/4387/SPC/ Zestril+Tablets/. Published 2010.

52. Varbella F, Musumeci G, Marchese A, Tarantini G. Ottimizzazione della terapia antiaggregante nelle sindromi coronariche acute. Edizioni Minerva Medica; 2011:1-10.

53. Bibbins-Domingo K. Aspirin use for the primary prevention of cardiovascular disease and colorectal cancer: U.S. Preventive Services Task Force Recommendation Statement. Ann Intern Med. 2016;164(12):836-845. doi:10.7326/m16-0577.

54. Thun MJ, Jacobs EJ, Patrono C. The role of aspirin in cancer prevention. Nat Rev Clin Oncol. 2012;9(5):259-267. doi:10.1038/nrclinonc.2011.199.

55. Elwood PC, Morgan G, Pickering JE, et al. Aspirin in the treatment of cancer: reductions in metastatic spread and in mortality: a systematic review and meta-analyses of published studies. PLoS One. 2016;11(4):e0152402. doi:10.1371/ journal.pone.0152402.

56. Rothwell PM, Wilson M, Price JF, Belch JF, Meade TW, Mehta Z. Effect of daily aspirin on risk of cancer metastasis: a study of incident cancers during randomised controlled trials. Lancet. 2012;379(9826):1591-1601. doi:10.1016/s01406736(12)60209-8.

57. Rothwell PM, Price JF, Fowkes FG, et al. Short-term effects of daily aspirin on cancer incidence, mortality, and non-vascular death: analysis of the time course of risks and benefits in 51 randomised controlled trials. Lancet. 2012;379(9826):160212. doi:10.1016/s0140-6736(11)61720-0.

58. Dewilde WJ, Oirbans T, Verheugt FW, et al. Use of clopidogrel with or without aspirin in patients taking oral anticoagulant therapy and undergoing percutaneous coronary intervention: an open-label, randomised, controlled trial. Lancet. 2013;381(9872):1107-15. doi:10.1016/s01406736(12)62177-1.

59. Roffi M, Patrono C, Collet JP, et al. 2015 ESC Guidelines for the management of acute coronary syndromes in patients presenting without persistent ST-segment elevation: Task Force for the Management of Acute Coronary Syndromes in Patients Presenting without Persistent ST-Segment Elevation of the European Society of Cardiology (ESC). Eur Heart J. 2016;37(3):267-315. doi:10.1093/eurheartj/ehv320.

60. CAPRIE Steering Committee. A randomised, blinded, trial of clopidogrel versus aspirin in patients at risk of ischaemic events (CAPRIE). Lancet. 1996;348(9038):1329-139. doi:10.1016/ S0140-6736(96)09457-3.

61. Johnston SC, Amarenco P, Albers GW, et al. Ticagrelor versus aspirin in acute stroke or transient ischemic attack. N Engl J Med. 2016;375(1):35-43. doi:10.1056/NEJMoa1603060. 